Supplement of The Cryosphere Discuss., 8, 3525-3561, 2014

http://www.the-cryosphere-discuss.net/8/3525/2014/

doi:10.5194/tcd-8-3525-2014-supplement

(C) Author(s) 2014. CC Attribution 3.0 License.

(c) (1)
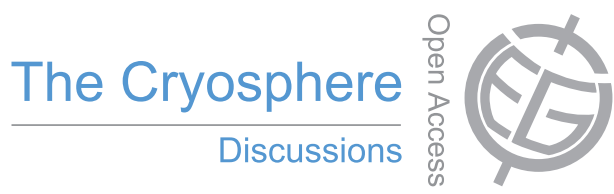

Supplement of

\title{
Are seasonal calving dynamics forced by buttressing from ice mélange or undercutting by melting? Outcomes from full-Stokes simulations of Store Gletscher, West Greenland
}

\author{
J. Todd and P. Christoffersen
}

Correspondence to: J. Todd (jat71@cam.ac.uk) 


\section{Lateral Convergence}

As part of the present study, we developed an algorithm to artificially add mass to the 2D flowline model to account for the out-of-plane effect of lateral convergence. Stokes flow of an incompressible fluid requires that there is no divergence in the flow field:

$$
\nabla \cdot \vec{u}=0
$$

However, if one were to observe a 2D slice down a glacier flowline, one would find that Equation S1 does not hold and, instead, a divergence term appears wherever there is a change in the neglected third dimension, i.e. narrowing or widening of the fjord. For any arbitrary area of the 2D domain, in our case, an element, any proportional change in glacier width will result in a change in element area/flux of equal proportion, but opposite sign:

$$
\frac{d A}{d x} A^{-1}=-\frac{d W}{d x} W^{-1}
$$

where $\mathrm{A}$ and $\mathrm{W}$ are element area and width respectively. To find the rate of area change, we multiply both sides by the x-velocity of the element:

$$
\frac{d A}{d x} \frac{d x}{d t} A^{-1}=-\frac{d W}{d x} W^{-1} U_{x}
$$

and rearrange:

$$
\frac{d A}{d t}=-\frac{d W}{d x} W^{-1} U_{x} A
$$

Finally, to implement this artificial convergence term in the Elmer/Ice Stokes solver, we insert Equation S4 into Equation S1:

$$
\nabla \cdot \vec{u}=-\frac{d W}{d x} W^{-1} u_{x} A
$$

\section{Remeshing after calving}

Like most numerical methods for modelling the dynamics of ice, the Finite Element Method (FEM) adopts a continuum mechanics approach, as opposed to attempting to resolve the interaction between individual ice crystals. This is an extremely useful modelling framework, but it is inherently incapable of 
dealing with fracture and the separation of icebergs. As such, it was necessary to develop a remeshing and interpolation algorithm to facilitate the retreat of the terminus following calving.

We adopt a lagrangian approach to changes in terminus position; as the terminus of the glacier advances in the $\mathrm{x}$-coordinate direction, the mesh travels with it. However, when a calving event occurs, it is inappropriate to simply 'push back' the terminus, as this has two non-physical consequences: first, the upper and bottom surfaces are compressed in the x-direction, and secondly, nodal values of the various model variables are unchanged, and so are essentially advected upstream. Overcoming these mesh-related issues represented a key challenge to this investigation.

In order to preserve the surface and basal topography of the mesh following calving, we treat the vertical $(\mathrm{z})$ coordinate as a variable $(H)$ which is then interpolated between meshes. Let $\Omega_{1}$ be the glacier body before calving and $\Omega_{2}$ the post-calving body. Each body has upper and lower boundary conditions, $\partial \Omega_{\text {surf }}$ and $\partial \Omega_{\text {bed }}$ respectively (Fig. S1).

In $\Omega_{1}$, we need only define the height variable $(H)$ on $\partial \Omega_{\text {surf }}$ and $\partial \Omega_{\text {bed }}$ :

$$
\begin{gathered}
\left.H\right|_{\partial \Omega_{\text {bed }}}=z \\
\left.H\right|_{\partial \Omega_{\text {surf }}}=z
\end{gathered}
$$

Next, we define the nodes in $\Omega_{2}$ by translating the $\Omega_{1}$ nodes in the xdirection to account for calving. We now carry out a reduced dimension interpolation of $H$ on both $\partial \Omega_{\text {surf }}$ and $\partial \Omega_{\text {bed }}$ to find the new heights of the surface and basal boundary conditions. In the present $2 \mathrm{D}$ problem, this is a line search in the $\mathrm{x}$-direction, whereas for a $3 \mathrm{D}$ case, this would be a $2 \mathrm{D}$ interpolation problem in the $\mathrm{x}-\mathrm{y}$ plane.

Once the updated surface and bed heights $(H)$ have been interpolated from $\Omega_{1}$, the height of the internal nodes can be found by solving the Laplace Equation:

$$
\Delta H=0
$$

Finally, it remains to update the post-calving mesh:

$$
\left.z\right|_{\Omega_{2}}=H
$$


Once the correct post-calving boundary and node positions have been obtained as described above, variables such as the stress and velocity vectors, as well as scalar fields such as glacier width, are simply interpolated from $\Omega_{1}$ to $\Omega_{2}$.

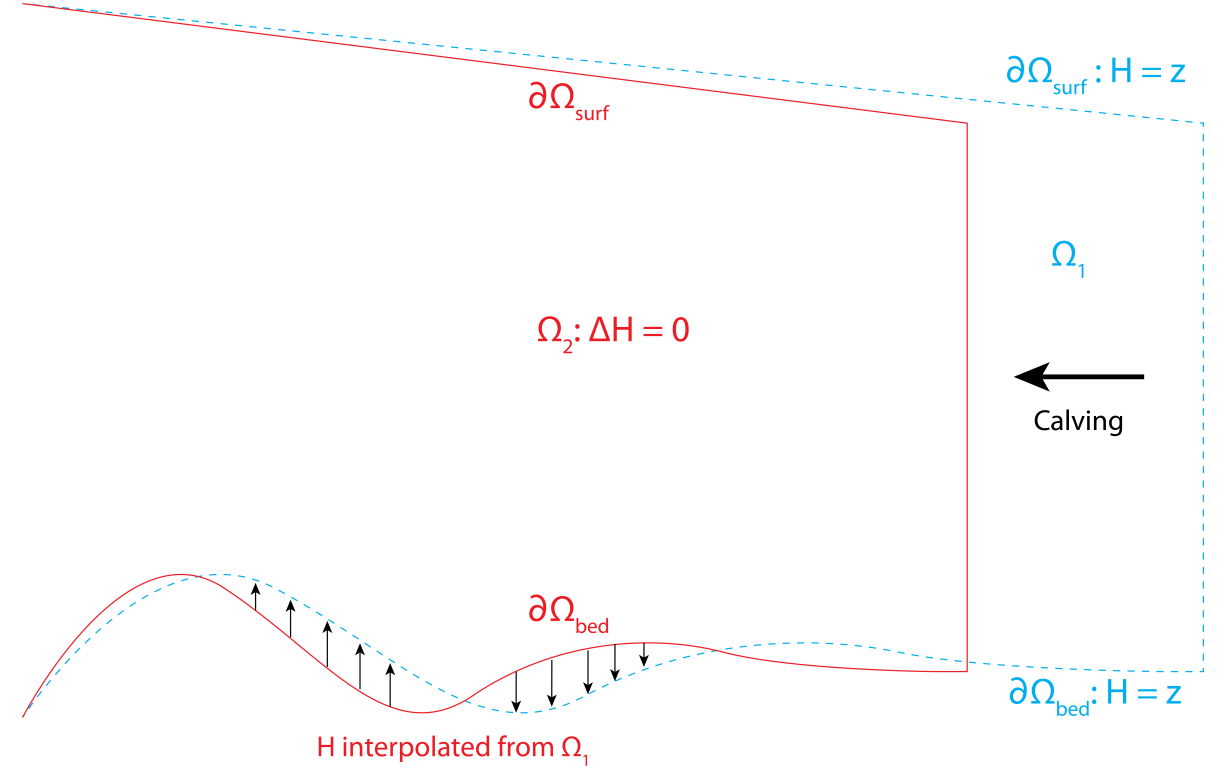

Figure S1: Description of the remeshing problem. Blue and red represent the pre- and post-calving geometry, respectively. 